Supplement of

\title{
Regional-scale modelling for the assessment of atmospheric particulate matter concentrations at rural background locations in Europe
}

Goran Gašparac et al.

Correspondence to: Goran Gašparac (goran.gasparac@ crocontrol.hr)

The copyright of individual parts of the supplement might differ from the CC BY 4.0 License. 
Table S1. Intercomparison of applied statistical measures (BIAS,IOA, r, RMSE, NMSE $E_{\text {sys }}$, $N M S E_{\text {unsys }}$ ) with minimum, median and maximum values, between measured (310 rural background stations from AirBase database, https://www.eea.europa.eu/data-andmaps/data/airbase-the-european-air-quality-database-7, and the EU-PHARE project) and modelled $\left(\overline{P M_{10}}\right)_{d}$ with the WRF-Chem and EMEP models during November 2011 with respect to the station height (same as Fig 8).

\begin{tabular}{|c|c|c|c|c|c|c|c|}
\hline & & & RF-Chem & & & EMEP & \\
\hline & $\begin{array}{l}\text { Station } \\
\text { Height }\end{array}$ & MIN & MEDIAN & MAX & MIN & MEDIAN & MAX \\
\hline & Sea-level & -86 & -44 & 2 & -68 & -26 & 47 \\
\hline$B I A S$ & Elevated & -91 & -54 & 100 & -80 & -29 & 132 \\
\hline & Mountain & -91 & -33 & 196 & -76 & 13 & 226 \\
\hline & Sea-level & 0.31 & 0.53 & 0.85 & 0.26 & 0.57 & 0.89 \\
\hline$I O A$ & Elevated & 0.19 & 0.44 & 0.88 & 0.06 & 0.47 & 0.86 \\
\hline & Mountain & 0.12 & 0.40 & 0.82 & 0.11 & 0.38 & 0.76 \\
\hline & Sea-level & 0.02 & 0.39 & 0.87 & 0.02 & 0.48 & 0.87 \\
\hline $\boldsymbol{r}$ & Elevated & 0.00 & 0.21 & 0.88 & 0.00 & 0.28 & 0.85 \\
\hline & Mountain & 0.01 & 0.19 & 0.82 & 0.00 & 0.24 & 0.75 \\
\hline & Sea-level & 6.89 & 20.75 & 60.84 & 5.02 & 17.36 & 50.17 \\
\hline$R M S E$ & Elevated & 4.16 & 18.99 & 114.67 & 3.46 & 15.32 & 111.01 \\
\hline & Mountain & 2.21 & 12.74 & 36.59 & 3.04 & 13.24 & 34.01 \\
\hline & Sea-level & $6.1 e-6$ & 0.34 & 5.53 & $7.3 e-6$ & 0.09 & 1.41 \\
\hline$N M S E_{s y s}$ & Elevated & $7.8 e-5$ & 0.62 & 9.30 & 0.0 & 0.18 & 3.32 \\
\hline & Mountain & 0.0 & 0.35 & 9.36 & $9.7 e-5$ & 0.21 & 2.38 \\
\hline & Sea-level & -0.74 & 0.41 & 1.74 & -0.26 & 0.26 & 0.86 \\
\hline$N M S E_{\text {unsys }}$ & Elevated & 0.02 & 0.52 & 1.88 & 0.08 & 0.27 & 1.55 \\
\hline & Mountain & 0.17 & 0.66 & 2.40 & 0.08 & 0.40 & 1.55 \\
\hline
\end{tabular}


Table S2. The minimum, maximum and median values of $\left(\overline{P M_{10}}\right)_{d}$, $(\overline{m s l p})_{d},\left(\overline{t_{2 m}}\right)_{d},(\overline{p b l h})_{d},(\overline{w s})_{d}$ over the domain (Fig 1) for WRF-Chem model during both episodes.

\begin{tabular}{|c|c|c|c|c|c|c|c|c|c|c|c|c|c|c|c|}
\hline \multirow[b]{2}{*}{ Day } & \multicolumn{3}{|c|}{$\left(P M_{10}\right)_{d}$} & \multicolumn{3}{|c|}{$(\overline{m s l p})$} & \multicolumn{3}{|c|}{$\left(\overline{t_{2 m}}\right)_{d}$} & \multicolumn{3}{|c|}{$\overline{p b l h}$} & \multicolumn{3}{|c|}{$(\overline{W S})_{d}$} \\
\hline & MIN & MAX & MEDIAN & MIN & MAX & MEDIAN & MIN & MAX & MEDIAN & MIN & MAX & MEDIAN & MIN & MAX & MEDIAN \\
\hline 5 & 0.17 & 34.76 & 4.17 & 998.60 & $1,025.76$ & $1,011.24$ & 0.78 & 21.81 & 11.93 & 51.14 & $1,581.14$ & 404.68 & 0.03 & 36.34 & 5.59 \\
\hline 6 & 0.18 & 63.55 & 3.83 & 999.13 & $1,031.99$ & $1,018.62$ & -0.46 & 21.48 & 11.48 & 45.80 & $1,633.84$ & 419.54 & 0.11 & 34.02 & 6.72 \\
\hline 7 & 0.19 & 47.24 & 5.39 & $1,004.90$ & $1,033.89$ & $1,019.55$ & -2.01 & 21.40 & 10.65 & 46.06 & $1,616.29$ & 319.23 & 0.09 & 25.03 & 5.38 \\
\hline 8 & 0.19 & 57.69 & 8.15 & $1,006.33$ & $1,030.76$ & $1,017.09$ & -1.11 & 21.31 & 10.74 & 34.41 & $1,450.01$ & 297.31 & 0.04 & 27.08 & 4.52 \\
\hline 9 & 0.19 & 59.39 & 7.74 & $1,001.21$ & $1,028.03$ & $1,018.95$ & -4.70 & 21.27 & 11.36 & 41.57 & $1,250.24$ & 284.93 & 0.02 & 19.89 & 3.87 \\
\hline 12 & 0.19 & 58.43 & 6.98 & $1,008.10$ & $1,040.86$ & $1,027.97$ & -9.75 & 20.69 & 11.02 & 40.80 & $1,525.72$ & 330.18 & 0.06 & 25.97 & 5.66 \\
\hline 13 & 0.19 & 81.32 & 7.05 & $1,007.63$ & $1,038.40$ & $1,030.70$ & -8.80 & 21.11 & 10.74 & 27.44 & $1,899.58$ & 299.69 & 0.01 & 27.17 & 5.32 \\
\hline 14 & 0.20 & 81.05 & 8.12 & $1,005.11$ & $1,031.63$ & $1,026.08$ & -8.94 & 20.67 & 9.24 & 26.38 & $1,955.07$ & 260.41 & 0.01 & 26.52 & 4.70 \\
\hline 15 & 0.19 & 70.83 & 9.37 & $1,007.43$ & $1,029.09$ & $1,021.05$ & -10.03 & 19.87 & 8.39 & 29.37 & $1,708.43$ & 299.64 & 0.00 & 21.72 & 4.39 \\
\hline AVG & 0.19 & 62.04 & 6.91 & $1,004.77$ & $1,031.93$ & $1,021.18$ & -5.54 & 20.89 & 10.34 & 38.27 & $1,612.29$ & 322.16 & 0.04 & 26.35 & 5.00 \\
\hline
\end{tabular}

Table S3. The minimum, maximum and median values of $\left(\overline{P M_{10}}\right)_{d}$, $(\overline{m s l p})_{d},\left(\overline{t_{2 m}}\right)_{d},(\overline{p b l h})_{d},(\overline{w s})_{d}$ over the domain (Fig 1) for EMEP model during both episodes.

\begin{tabular}{|c|c|c|c|c|c|c|c|c|c|c|c|c|c|c|c|}
\hline \multirow[b]{2}{*}{ Day } & \multicolumn{3}{|c|}{$\left.\overline{P M_{10}}\right)_{d}$} & \multicolumn{3}{|c|}{$m s(p)$} & \multicolumn{3}{|c|}{$\left(\overline{t_{2 m}}\right)$} & \multicolumn{3}{|c|}{$\overline{p b l h}$} & \multicolumn{3}{|c|}{$(\overline{W S})_{d}$} \\
\hline & MIN & MAX & MEDIAN & MIN & MAX & MEDIAN & MIN & MAX & MEDIAN & MIN & MAX & MEDIAN & MIN & MAX & MEDIAN \\
\hline 5 & 1.10 & 82.55 & 12.78 & $1,000.20$ & $1,026.08$ & $1,011.79$ & 0.27 & 20.82 & 11.42 & 134.42 & $1,628.13$ & 614.25 & 0.04 & 14.81 & 4.64 \\
\hline 6 & 1.13 & 110.09 & 11.76 & $1,003.34$ & $1,030.98$ & $1,018.15$ & 0.31 & 21.13 & 10.94 & 132.20 & $1,604.03$ & 609.60 & 0.18 & 16.11 & 4.99 \\
\hline 7 & 1.19 & 95.88 & 12.37 & $1,006.36$ & $1,033.38$ & $1,019.29$ & -0.82 & 20.72 & 10.15 & 153.99 & $1,406.29$ & 499.97 & 0.04 & 15.70 & 3.58 \\
\hline 8 & 1.16 & 73.12 & 14.54 & $1,005.62$ & $1,029.38$ & $1,017.84$ & -0.63 & 20.44 & 10.43 & 137.09 & $1,244.23$ & 444.85 & 0.03 & 14.09 & 3.10 \\
\hline 9 & 1.25 & 78.60 & 12.54 & $1,001.58$ & $1,028.91$ & $1,019.23$ & -1.35 & 20.22 & 11.28 & 152.47 & $1,163.56$ & 392.36 & 0.04 & 12.74 & 2.63 \\
\hline 12 & 2.64 & 81.96 & 14.91 & $1,007.41$ & $1,040.12$ & $1,026.67$ & -3.68 & 20.02 & 11.36 & 122.74 & $1,732.04$ & 503.42 & 0.04 & 15.24 & 3.67 \\
\hline 13 & 2.40 & 72.48 & 15.43 & $1,008.17$ & $1,038.18$ & $1,028.08$ & -4.57 & 19.74 & 10.95 & 122.30 & $1,843.41$ & 495.59 & 0.01 & 17.28 & 4.08 \\
\hline 14 & 1.61 & 97.84 & 15.35 & $1,005.91$ & $1,030.13$ & $1,023.16$ & -4.76 & 20.01 & 9.43 & 126.60 & $1,778.65$ & 503.42 & 0.05 & 16.77 & 3.66 \\
\hline 15 & 1.23 & 72.21 & 12.65 & $1,008.09$ & $1,028.25$ & $1,018.74$ & -3.31 & 19.22 & 8.34 & 147.04 & $1,798.95$ & 487.96 & 0.03 & 14.32 & 3.08 \\
\hline 16 & 1.36 & 79.80 & 12.36 & $1,008.81$ & $1,029.01$ & $1,020.32$ & -4.58 & 19.27 & 8.44 & 147.33 & $1,658.80$ & 423.41 & 0.02 & 11.86 & 2.58 \\
\hline AVG & 1.51 & 84.45 & 13.47 & 1005.55 & 1031.44 & 1020.33 & -2.31 & 20.16 & 10.27 & 137.62 & 1585.81 & 497.48 & 0.05 & 14.89 & 3.60 \\
\hline
\end{tabular}




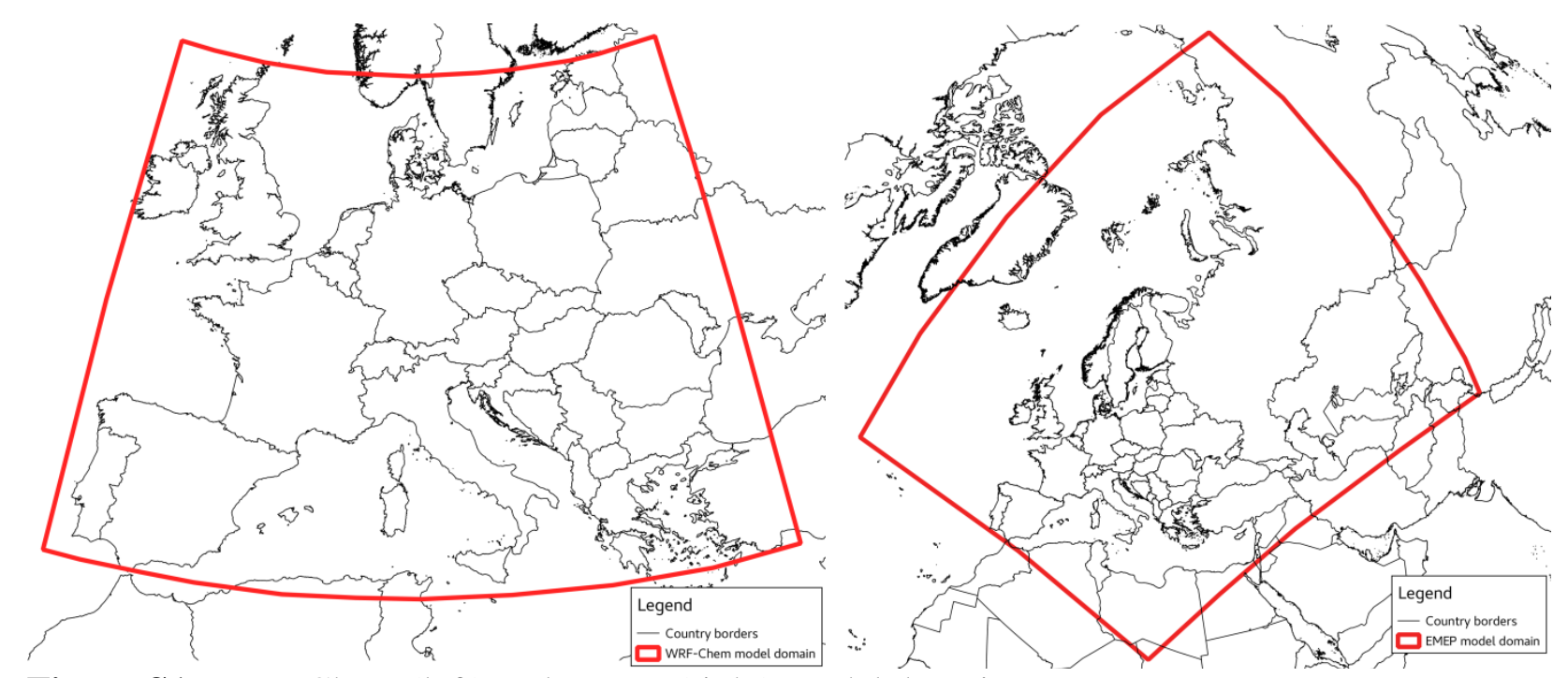

Figure S1. WRF-Chem (left) and EMEP (right) model domains.

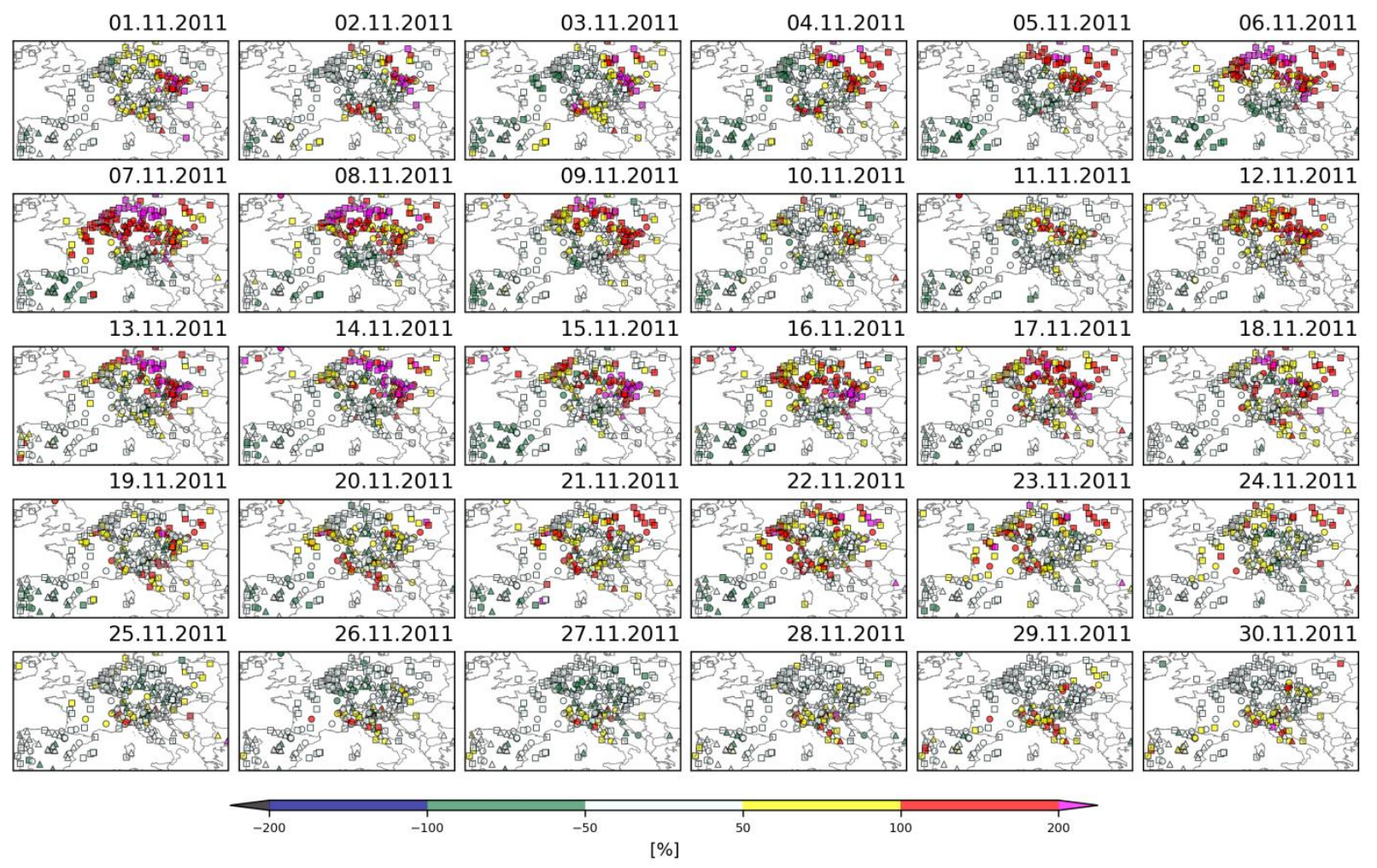

Figure S2. The difference $(D F)$ between $\left(\overline{P M_{10}}\right)_{d}$ and $\left(\overline{P M_{10}}\right)_{a}$ concentrations (equation 10) over Europe during November 2011. 


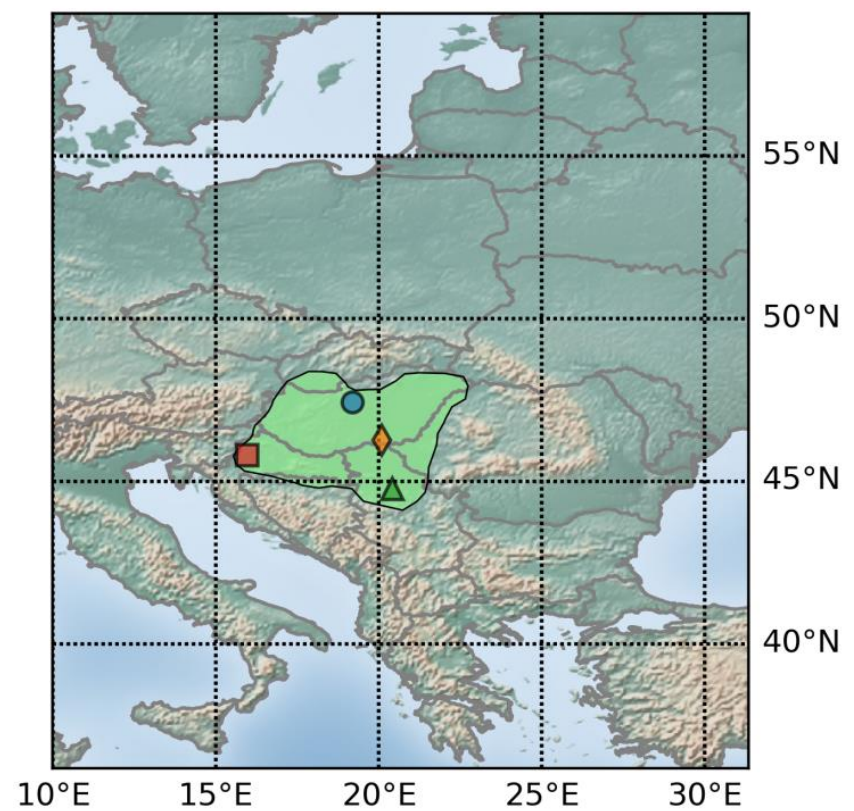

Figure S3. Pannonian basin (green area) with the location of measurement stations $\boldsymbol{\Delta}$ Belgrade, •-Budapest, - Szeged, - Zagreb. 

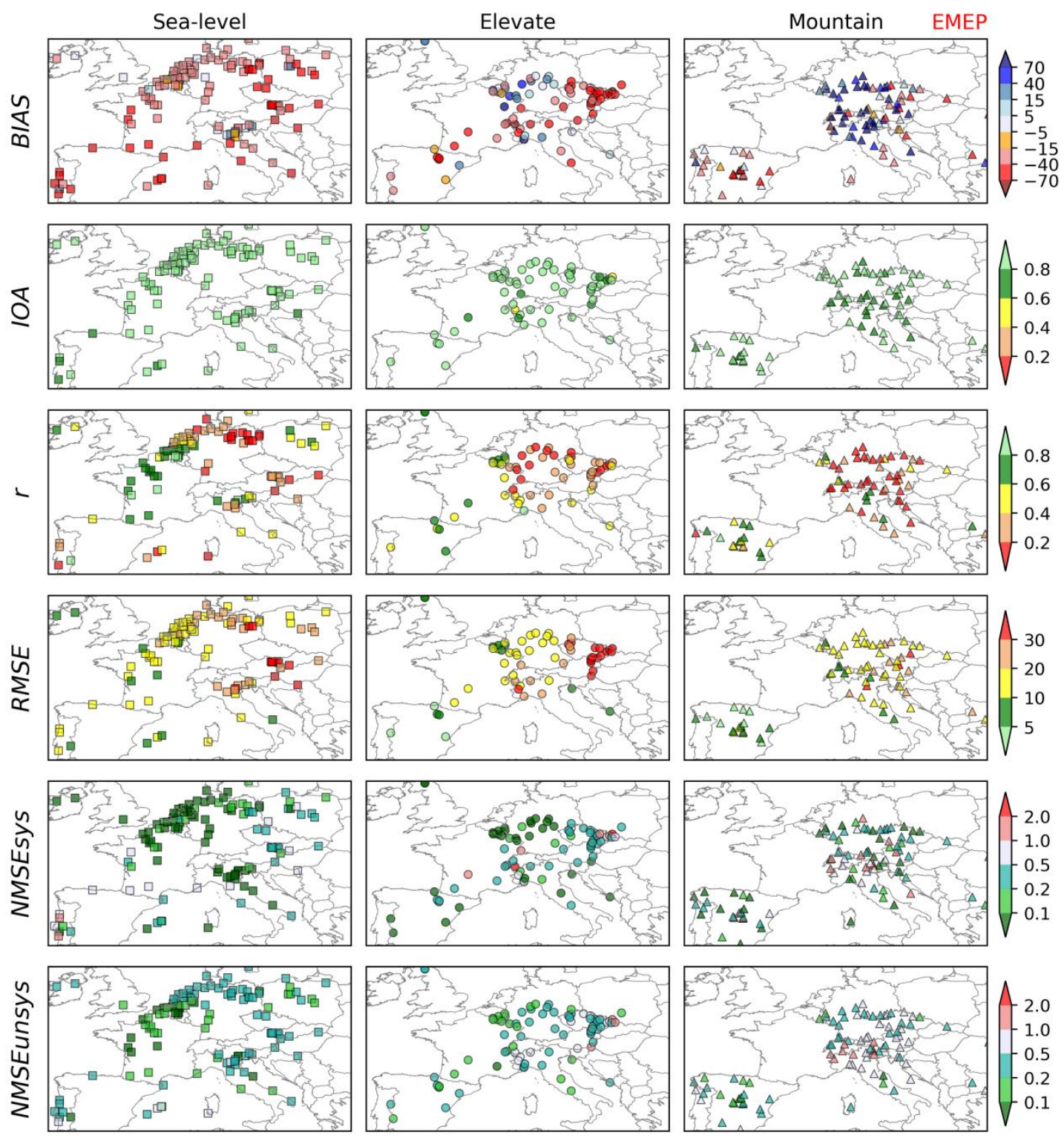

Figure S4. Comparison of statistical parameters between measured (310 rural background stations from AirBase database, https://www.eea.europa.eu/data-and-maps/data/airbase-theeuropean-air-quality-database-7, and the EU-PHARE project) and modelled $\left(\overline{P M_{10}}\right)_{d}$ with the EMEP model during a one-month period (November 2011) with respect to the station height: BIAS, IOA, $r$, RMSE, NMSEunsys, NMSEsys. 

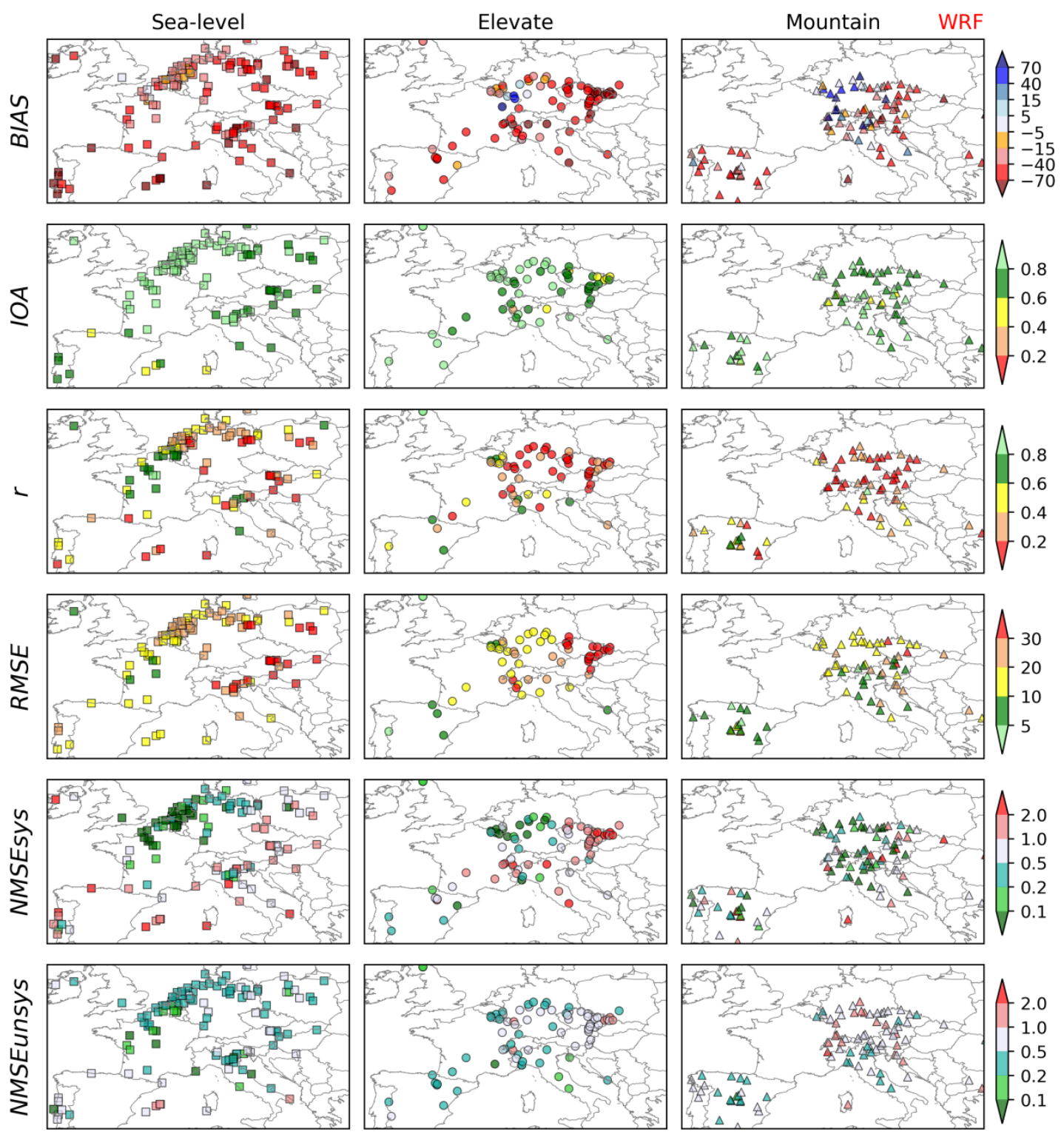

Figure S5. Same as Fig S4, but for WRF-Chem model. 

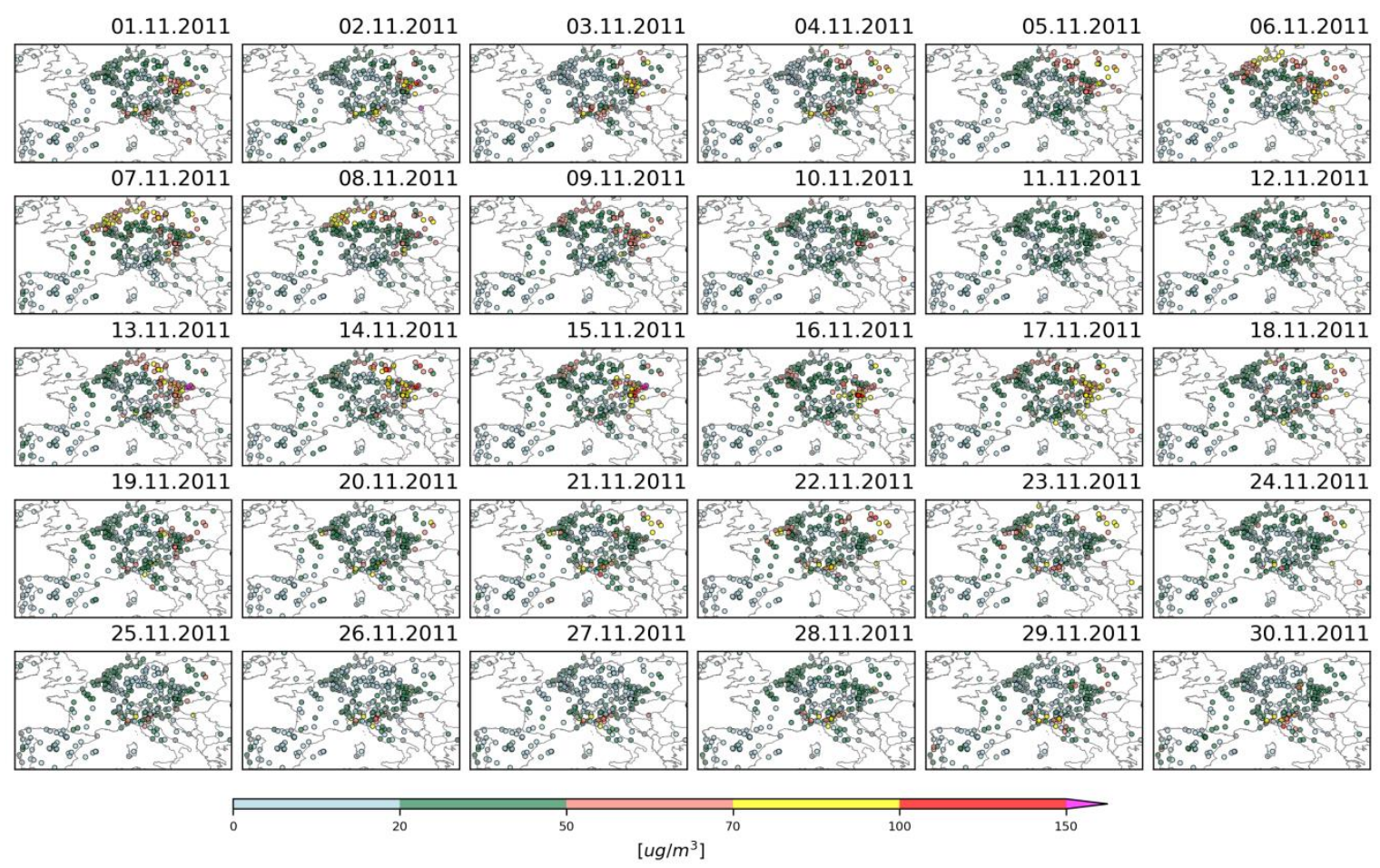

Figure S6. $\left(\overline{P M_{10}}\right)_{d}$ for all rural background stations within the domain during November 2011. 

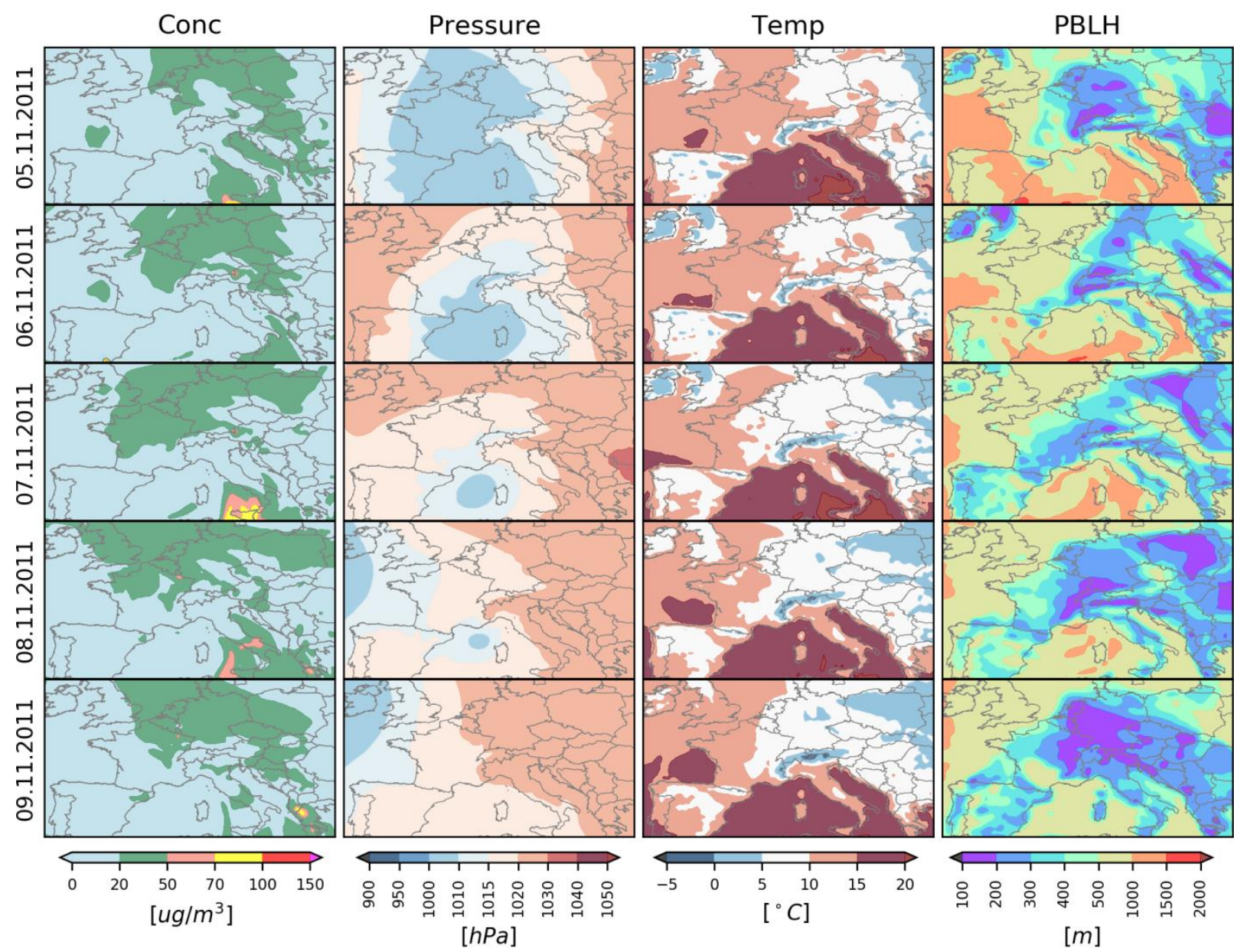

Figure S7. Modelled $\left(\overline{P M_{10}}\right)_{d}$ as Conc, and $(\overline{m s l p})_{d}$ as Pressure, $\left(t_{2 m}\right)_{d}$ as Temp and $(\overline{p b l h})_{d}$ as $P B L H$ during the first high pollution episode (the EMEP model). 


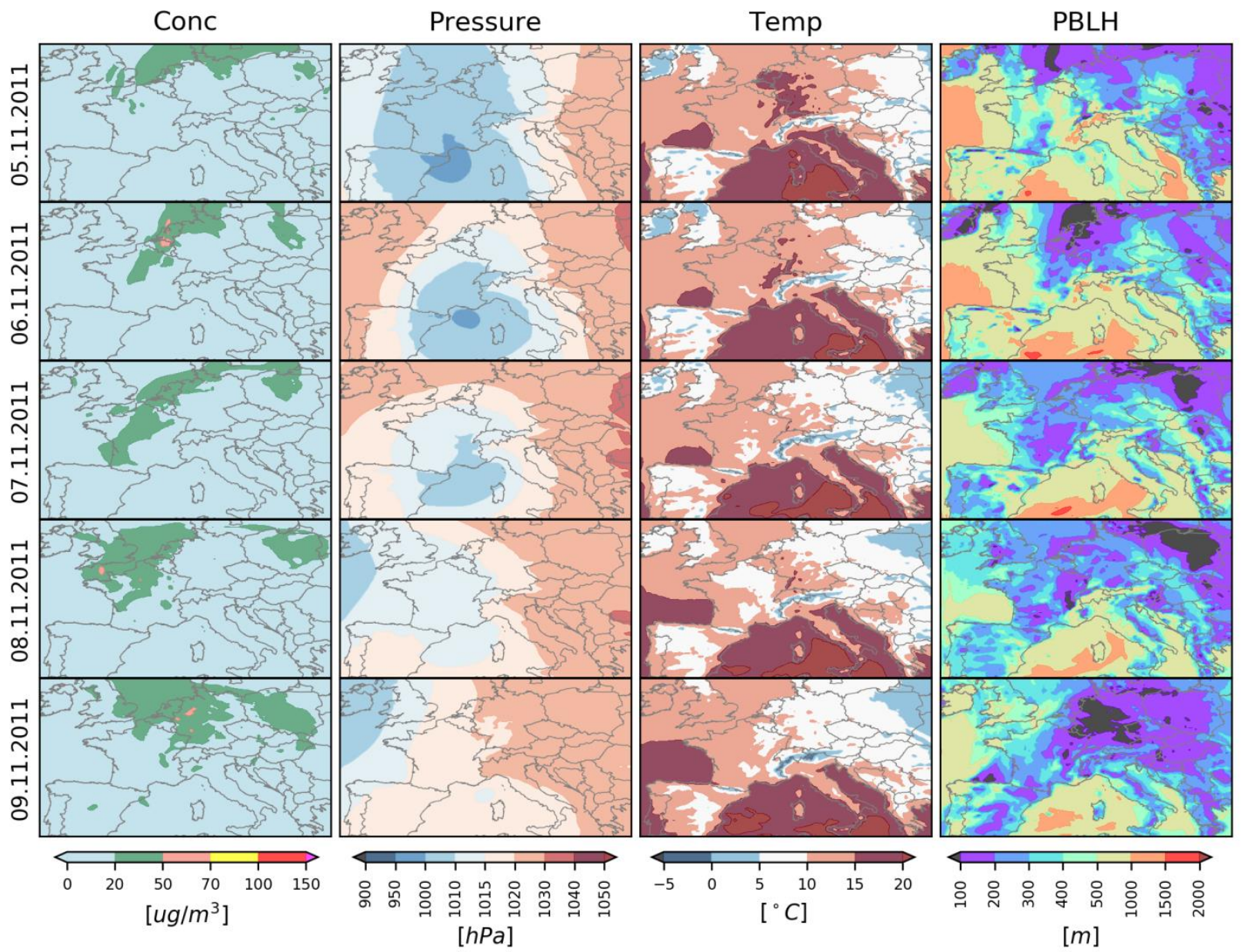

Figure S8. Same as Fig S7 but for the WRF-Chem model. 


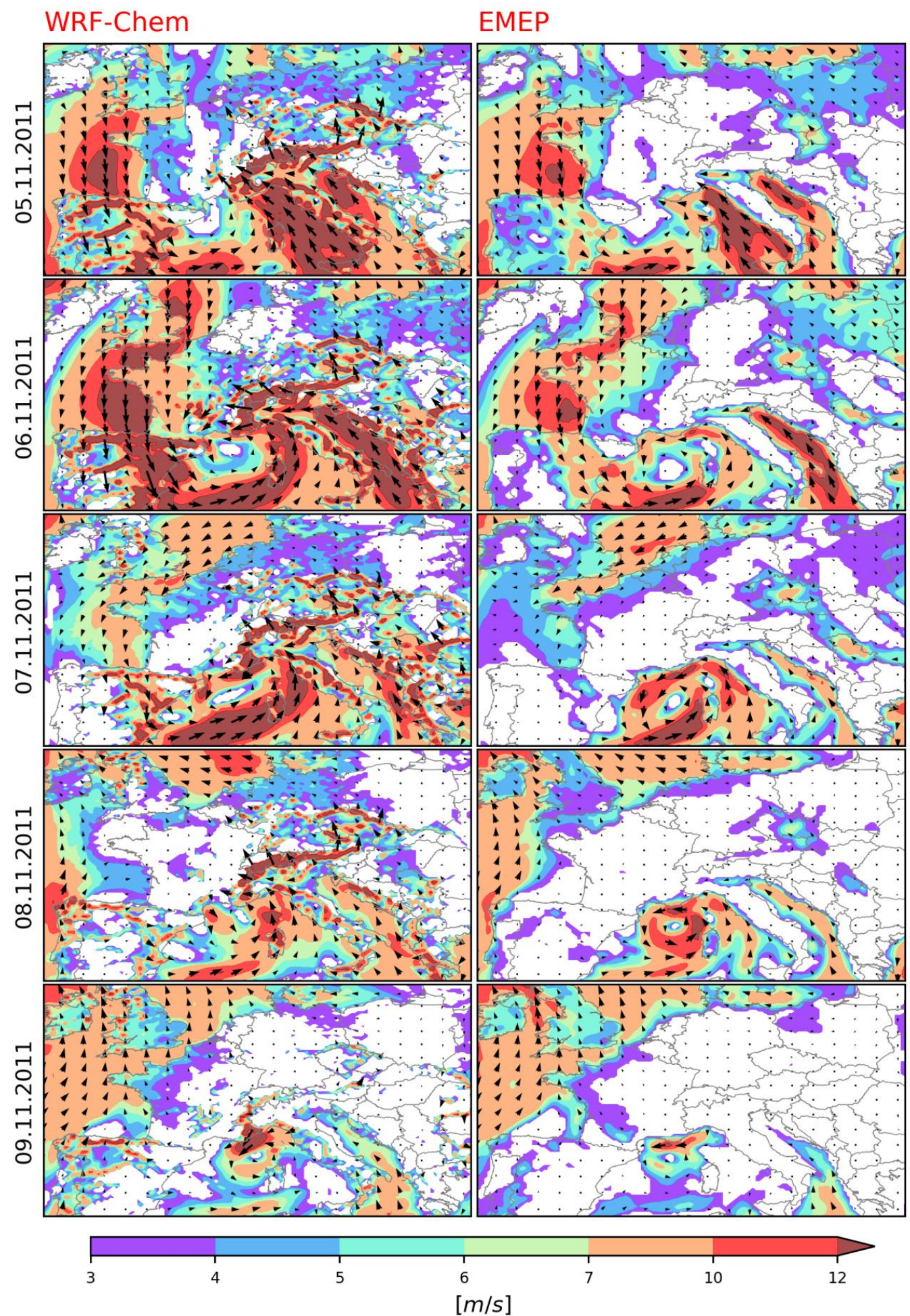

Figure S9 Modelled daily averaged wind speed and direction during the first high pollution episode for the WRF-Chem (left) and EMEP models (right). 

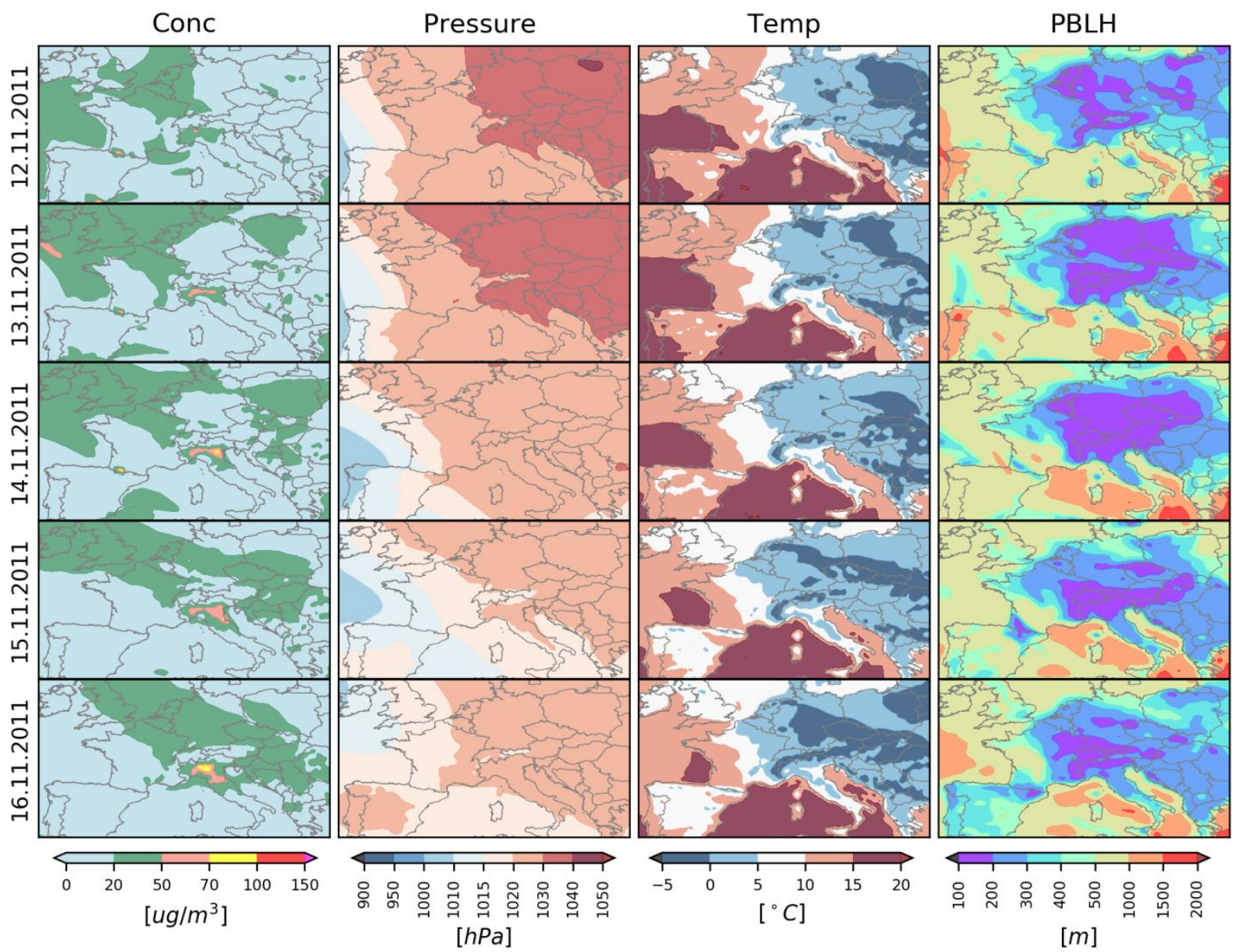

Figure S10. Modelled $\left(\overline{P M_{10}}\right)_{d}$ as Conc, and $(\overline{m s l p})_{d}$ as Pressure, $\left(\overline{t_{2 m}}\right)_{d}$ as Temp and $(\overline{p b l h})_{d}$ as $P B L H$ during the second high pollution episode (the EMEP model). 

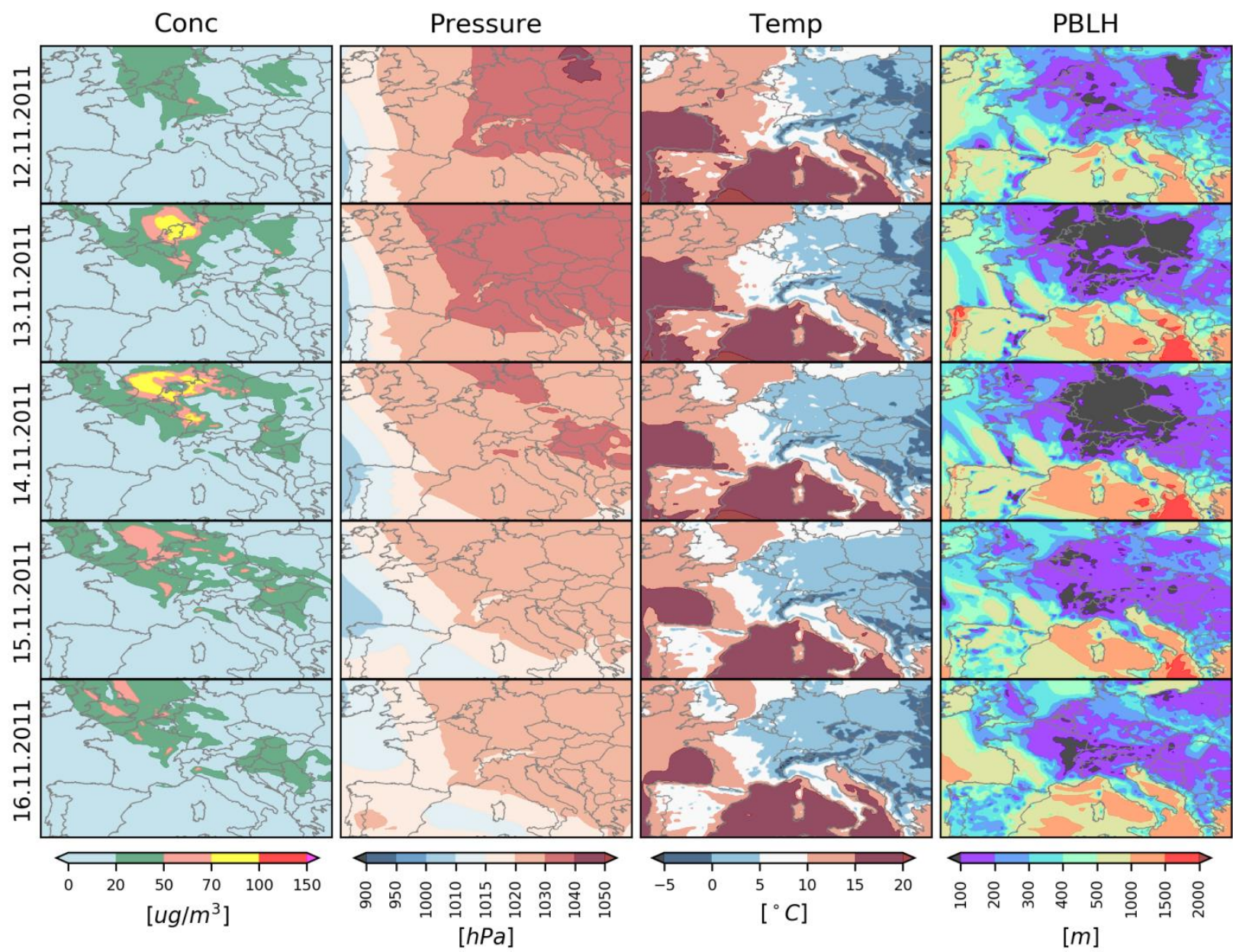

Figure S11. Same as Fig S10 but for the WRF-Chem model. 


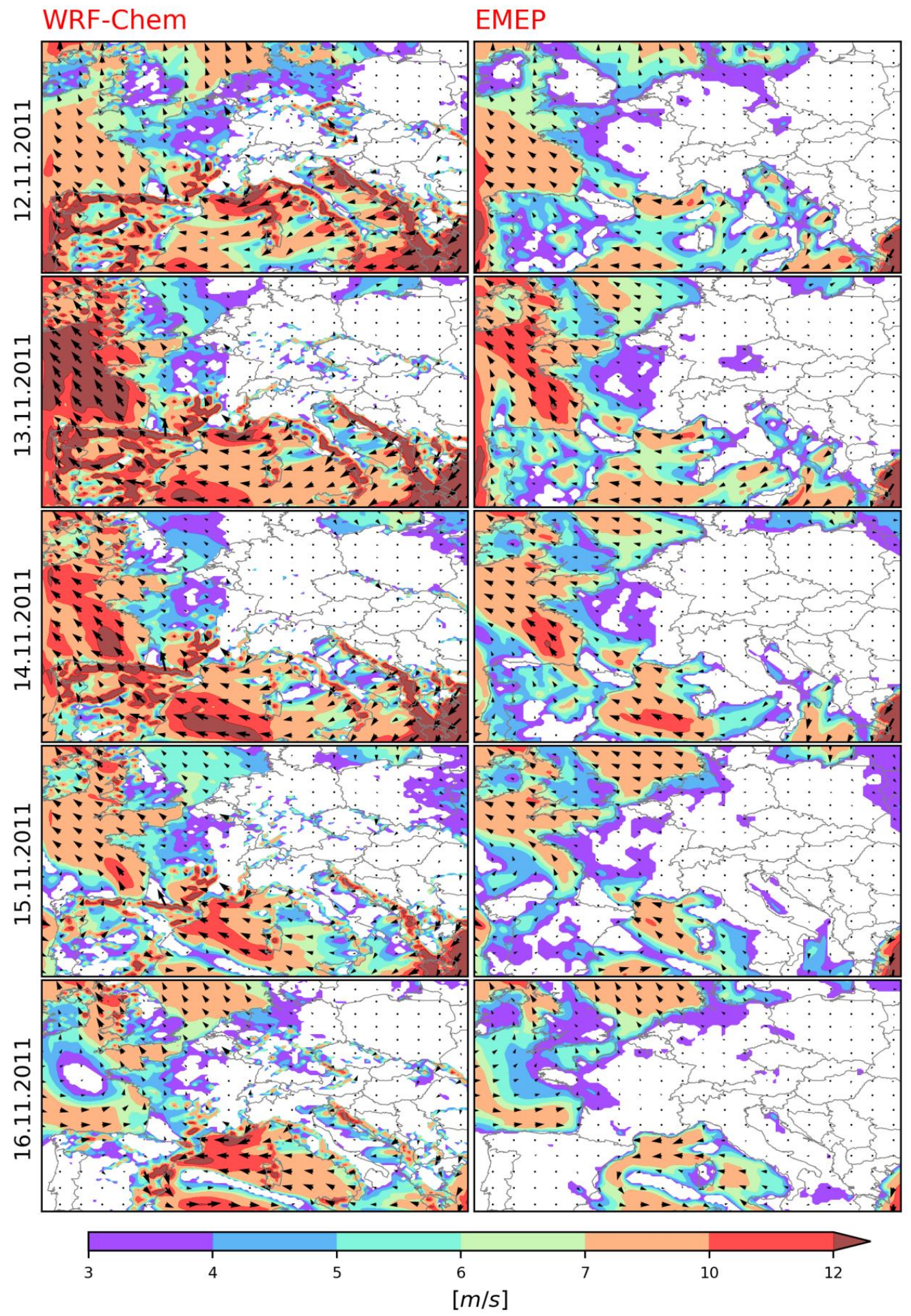

Figure S12. Modelled daily averaged wind speed and direction during the second high pollution episode for the WRF-Chem (left) and EMEP models (right). 


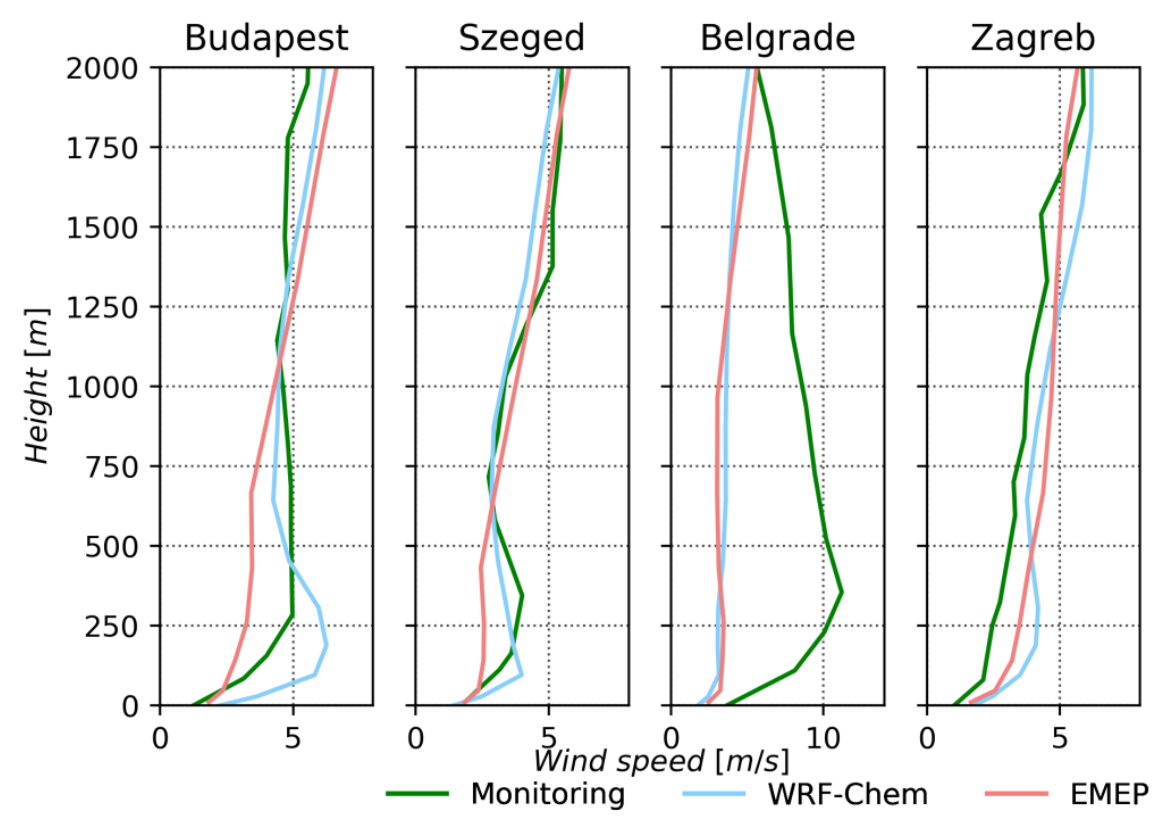

Figure S13. Comparison of the modelled averaged vertical profile for wind speed and soundings up to $2 \mathrm{~km}$ during the second high pollution episode (source: http://www.weather.uwyo.edu/upperair/sounding.html ; last access: 12 May 2020) 